\title{
Downregulation of FHL1 protein in glioma inhibits tumor growth through PI3K/AKT signaling
}

\author{
SAN-ZHONG LI ${ }^{1 *}$, YI-YANG HU ${ }^{2 *}$, JUN-LONG ZHAO ${ }^{2,3^{*}}$, JIAN ZANG $^{2,4}$, \\ ZHOU FEI ${ }^{1}$, HUA HAN ${ }^{2,3}$ and HONG-YAN QIN ${ }^{2}$ \\ Departments of ${ }^{1}$ Neurosurgery, ${ }^{2}$ Medical Genetics and Developmental Biology, ${ }^{3}$ Biochemistry and Molecular Biology and \\ ${ }^{4}$ Radiation Oncology, Xijing Hospital, Fourth Military Medical University, Xi'an, Shaanxi 710032, P.R. China
}

Received February 25, 2019; Accepted January 27, 2020

DOI: $10.3892 / 01.2020 .11476$

\begin{abstract}
Human four-and-a-half LIM domains protein 1 (FHL1) is a member of the FHL protein family, which serves an important role in multiple cellular events by interacting with transcription factors using its cysteine-rich zinc finger motifs. A previous study indicated that FHL1 was downregulated in several types of human cancer and served a role as a tumor suppressive gene. The overexpression of FHL1 inhibited tumor cell proliferation. However, to the best of our knowledge, there is no evidence to confirm whether FHL1 affected glioma growth, and the molecular mechanisms through which FHL1 represses tumor development remain unclear. In the present study, the expression level of FHL1 was determined using immunohistochemical staining in 114 tumor specimens from patients with glioma. The results indicated that FHL1 expression was negatively associated with the pathological grade of gliomas. Furthermore, Kaplan-Meier survival curves demonstrated that the patients with an increased FHL1 expression exhibited a significantly longer survival time, suggesting that FHL1 may be a prognostic marker for glioma. The protein level of FHL1 was relatively increased in the U251 glioma cell line compared with that in the U87 cell line. Therefore, FHL1 was knocked down in U251 by siRNA and overexpressed in U87, and it was identified that FHL1 significantly decreased the activation of PI3K/AKT signaling by interacting with AKT.
\end{abstract}

Correspondence to: Professor Hua Han, Department of Medical Genetics and Developmental Biology, Xijing Hospital, Fourth Military Medical University, 169 Chang-Le Xi Street, Xi'an, Shaanxi 710032, P.R. China

E-mail: huahan@fmmu.edu.cn

Professor Zhou Fei, Department of Neurosurgery, Xijing Hospital, Fourth Military Medical University, 169 Chang-Le Xi Street, Xi'an, Shaanxi 710032, P.R. China

E-mail: feizhou@fmmu.edu.cn

*Contributed equally

Key words: four-and-a-half LIM domains protein 1, glioma, proliferation, PI3K/AKT signaling
Further experiments verified that FHL1 inhibited the growth of gliomas in vivo by modulating PI3K/AKT signaling. In conclusion, the results of the present study demonstrated that FHL1 suppressed glioma development through PI3K/AKT signaling.

\section{Introduction}

Gliomas are the most aggressive, clinically intractable and unfortunately the most common type of primary tumors of the central nervous system. Following decades of advancements in the diagnosis and combined modality therapy of gliomas, the median survival following initial diagnosis of their most aggressive form, glioblastoma multiforme, WHO grade IV, remains at $\sim 14.6$ months, and the 5-year survival rate at $9.8 \%(1,2)$.

Human four-and-a-half LIM domains protein 1 (FHL1) is a member of the FHL protein family, and is characterized by its possession of four and a half LIM domains. The LIM domain was first identified during the isolation and identification of the linl gene in Caenorhabditis elegans, the isl-1 gene in rats, and the mec- 1 gene in C.elegans. It was named after the first letter of each gene mentioned above $(3,4)$. The LIM domain has protein-protein binding interfaces that possess cysteine-rich zinc finger motifs. Therefore, FHL1 serves an important role in cellular events, such as skeletal muscle growth, by interacting with transcription factors using its cysteine-rich zinc finger motifs (5).

Recently, FHL1 has been implicated in cancer. FHL1 is downregulated in several types of human cancer; the greatest levels of downregulation are observed in widely invasive and metastatic cases and associated with poor clinical prognosis (6-15). FHL1 overexpression suppresses cell growth through cyclin D1 and E and p27 in head and neck squamous cell carcinoma (16). FHL1 may also be converted from a tumor suppressor to a cell growth accelerator, when FHL1 is phosphorylated by cytosolic tyrosine kinase Src (17). However, its exact role and molecular mechanism in gliomas is poorly understood.

PI3K/AKT signaling controls diverse cellular functions in glioma, including proliferation, survival and migration $(18,19)$. When the activation of the PI3K/AKT pathway is inhibited, the levels of cell proliferation and cycle arrest are significantly decreased (20). The PI3K inhibitor combined with 
adenovirus-mediated PTEN could further suppress malignant glioma cell growth in vitro and in vivo (21). These studies indicated that PI3K/AKT signaling may be a key topic in the study of malignant glioma cell growth.

The present study explored the exact role of FHL1 in glioma and the molecular interactions between FHL1 and PI3K/AKT signaling. The results demonstrated that the expression of FHL1 was able to inhibit glioma cell growth in vitro and in vivo by modulating PI3K/AKT signaling through its interaction with AKT. FHL1 was also negatively associated with histological grades of glioma, suggesting that decreased FHL1 expression was associated with poor prognosis. In conclusion, the data from the present study provide evidence for a novel diagnostic and prognostic marker and a new target for glioma treatment in the future.

\section{Materials and methods}

Cell lines and animals. The human glioblastoma U251 cell line and a glioblastoma of unknown origin U87 cell line were purchased from the Cancer Institute of Fudan University. The U87 cell line was authenticated by STR profiling. All cells were cultured in Dulbecco's modified Eagle medium (Sigma-Aldrich; Merck KGaA )supplemented with $10 \%$ heat-inactivated fetal bovine serum (Thermo Fisher Scientific, Inc.) and $1 \%$ penicillin and streptomycin at $37^{\circ} \mathrm{C}$ in an incubator supplied with $5 \% \mathrm{CO}_{2}$.

Male BALB/cA-nu 6-week-old nude mice were purchased from the Shanghai Laboratory Animal Center, Chinese Academy of Sciences, and maintained under specific pathogen-free conditions. A total of 12 mice were randomly divided into two groups. The U87 cells stably expressing FHL1 or vehicle controls were injected subcutaneously into one side of the flank of each mouse with $5 \times 10^{5}$ cells in each group. A total of 7 days after the injection, the tumor size was monitored every 3 or 4 days using sliding caliper measurements, and tumor volumes were calculated according to the formula: Volume $=0.51 \mathrm{x}$ length $\mathrm{x}$ width $^{2}$. The mice in the present study were sacrificed after 24 day by cervical dislocation under intraperitoneal sodium pentobarbital anesthesia $(60 \mathrm{mg} / \mathrm{kg}$ ) to minimize discomfort. The tumors were excised for further experiments. No significant differences in the body weights of the experimental mice compared with those of mice prior to tumor cells inoculation were observed. The results demonstrated that the largest tumor volume observed in mice was $3.5 \times 10^{3} \mathrm{~mm}^{3}$.

The animal studies were approved by the Animal Experiment Administration Committee of the Fourth Military Medical University (approval no., 20190211), and in accordance with the recommendations of Guide for the Care and Use of Laboratory Animals prepared by the National Academy of Sciences and published by the National Institutes of Health (22).

Patients and tissue specimens. Frozen and paraffin-embedded glioma tissues $(\mathrm{n}=114)$ were obtained from the Department of Neurosurgery of Xijing Hospital, Fourth Military Medical University. Tumors were graded according to current World Health Organization guidelines (23). All patients involved in the study provided written informed consent for the use of their samples, and the protocols involvi0ng human samples were approved by the Ethics Committee of Xijing Hospital, Fourth Military Medical University (approval no., KY20183175-1). The clinical data of the patients are summarized in Table SI.

Immunohistochemistry. FHL1 expression was detected using immunohistochemical staining of the clinical samples, according to standard protocol. The glioma tissues were fixed within 30 min following resection using $10 \%$ formalin at room temperature for $24 \mathrm{~h}$. Block of tissue was cut into $5 \mu \mathrm{m}$-thick sections that were heated at $67^{\circ} \mathrm{C}$ for $120 \mathrm{~min}$. Sections were then deparaffinized twice with xylene for $5 \mathrm{~min}$, rehydrated with gradient ethanol $(100 \%$ for $5 \mathrm{~min}$ twice, $95 \%$ for $5 \mathrm{~min}$ twice, $90 \%$ for $5 \mathrm{~min}, 85 \%$ for $5 \mathrm{~min}$ and $80 \%$ for $5 \mathrm{~min}$ ) and washed with pure water for $1 \mathrm{~h}$. Sections were then pretreated with $3 \% \mathrm{H}_{2} \mathrm{O}_{2}$ for $15 \mathrm{~min}$ at $4^{\circ} \mathrm{C}$ to block endogenous peroxidase activity. Antigens were retrieved by pressure cooker treatment for $100 \mathrm{sec}$ in $0.01 \mathrm{mmol} / 1$ citrate buffer $(\mathrm{pH} 6)$. The primary antibody against FHL1 (cat. no. 10991-1-AP; Wuhan Sanying Biotechnology) was diluted at 1:150 and then incubated with tissue sections overnight at $4^{\circ} \mathrm{C}$. Normal rabbit $\operatorname{IgG}$ isotype was used as the negative control. The next day, the tissue sections were incubated with horseradish peroxidase (HRP)-conjugated secondary antibodies (Wuhan Boster Biological Technology, Ltd. cat. no. BM3894; 1:500) for $2 \mathrm{~h}$ at room temperature. Samples were visualized using a 3'-diaminobenzidine kit (OriGene Technologies, Inc.), and hematoxylin was used for counterstaining. Images were captured using a BX51 fluorescence microscope with a CCD camera (DP70; Olympus Corporation). Five randomized sights were selected (at least 1,000 tumor cells per slide) under a high-power microscope. The percentage of positive cells was calculated as 'positive cells' number/'all cells' number.

According to the percentage of FHL1 expression in total cells, tumors were classified into two categories: 0-20\% FHL1 positive staining in tumor cells, negative/low FHL1 expression; $>20 \%$ FHL1 positive staining in tumor cells, high FHL1 expression.

Western blot analysis. Western blot analysis was performed according to standard procedure as previously described (24). Cell or tissue lysates were obtained from clinical specimens and glioma cell lines by using lysis buffer (Beyotime Institute of Biotechnology) supplemented with complete protease inhibitor cocktail (Roche Diagnostics). The bicinchoninic acid protein assay kit (Thermo Fisher Scientific, Inc.) was used to measure protein concentration. Proteins $(25 \mu \mathrm{g})$ were separated by $10 \%$ SDS-PAGE and transferred onto polyvinylidene difluoride membranes. The membranes were blocked with 5\% non-fat dried milk in PBST for $2 \mathrm{~h}$ and incubated overnight with primary antibodies at an appropriate dilution at $4^{\circ} \mathrm{C}$. Following washing with PBST buffer, the membranes were then incubated with HRP-conjugated secondary antibodies for $1 \mathrm{~h}$ at room temperature and detected by an enhanced chemiluminescence detection system (Multi Science (Lianke) Biotech Co., Ltd). Primary antibodies including anti-FHL1 (1:1,200), anti-AKT (1:500), anti-phospho-AKT (p-AKT), which detects the endogenous levels of AKT with phosphorylation at threonine 308 (1:500; Signalway Antibody LLC), anti- $\beta$-actin $(1: 1,000$; Merck KGaA) and anti-FLAG (1:1,000; 
$A_{\text {I }}$
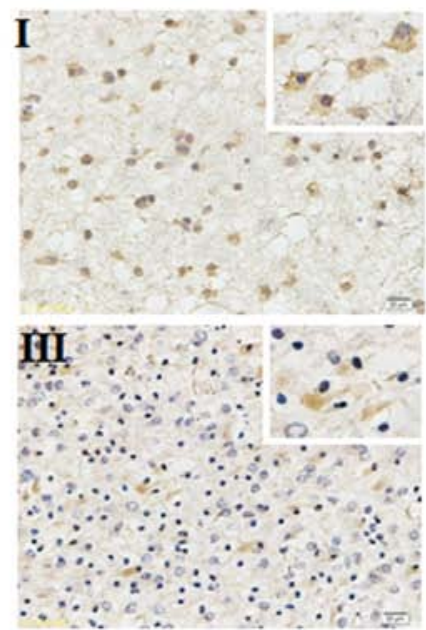
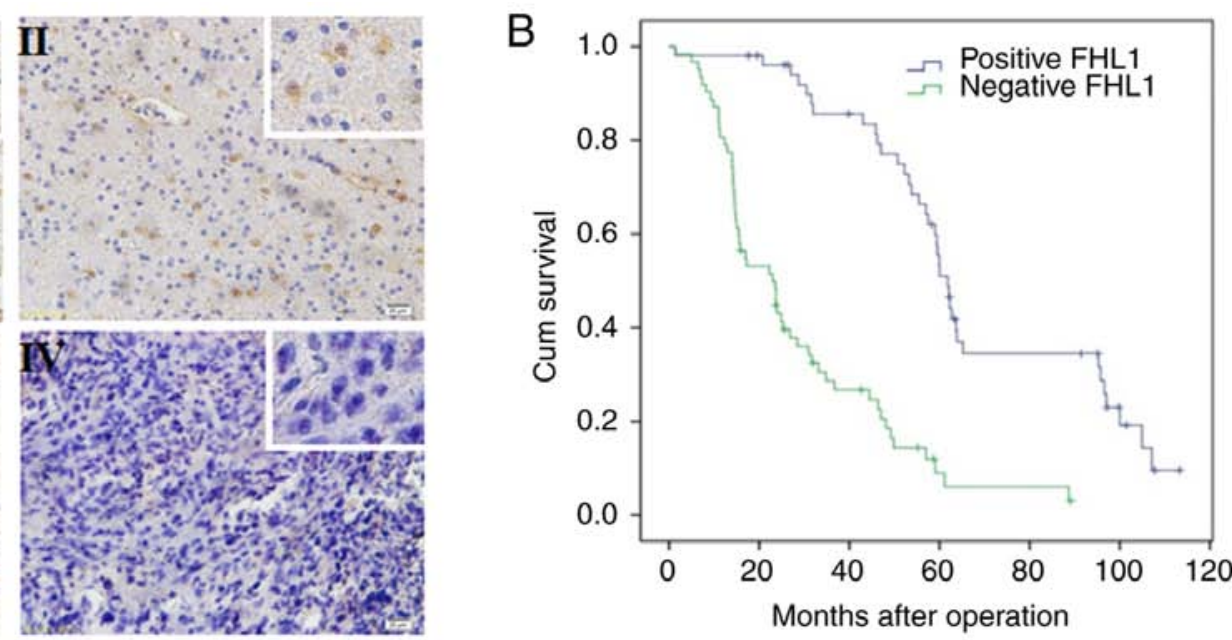

Figure 1. Expression of FHL1 in human glioma specimen is negatively associated with pathological grades and survival time. (A) The expression of FHL1 in different pathological grades of glioma specimen (grades I-IV) was determined by immunohistochemistry staining. Its expression was indicated by brown staining in the cytoplasm ( $\mathrm{n}=114$ ). Magnifications, $\mathrm{x} 100$ and x400 (white boxes). (B) Overall survival curves of patients with glioma with high or low/negative expression of FHL1 were calculated using the Kaplan-Meier method. The difference in survival time between the two groups was examined using a log-rank test, and low expression of FHL1 was identified to be significantly associated with poor prognosis. FHL1, four-and-a-half LIM domains 1.

Merck KGaA). As secondary antibodies, HRP-conjugated goat anti-rabbit IgG and goat anti-mouse IgG (Wuhan Boster Biological Technology, Ltd.) antibodies were used.

Cell transfection. Lentiviral vectors expressing FHL1 were constructed using lentiviral packaging systems as previously described (24). Briefly, a polymerase chain reaction (PCR) was performed by using the PCR kit (Takara Biotechnology Co., Ltd.) to amplify human FHL1 open read frame with human cDNA library as the template. The PCR thermocycling conditions were as follows: Initial denaturation at $95^{\circ} \mathrm{C}$ for $5 \mathrm{~min}$, 35 cycles of denaturation at $95^{\circ} \mathrm{C}$ for $30 \mathrm{sec}$, annealing at $58^{\circ} \mathrm{C}$ for $30 \mathrm{sec}$, elongation at $72^{\circ} \mathrm{C}$ for $45 \mathrm{sec}$ and final extension at $72^{\circ} \mathrm{C}$ for $5 \mathrm{~min}$. Human FHL1 fragment was inserted into the multiple cloning sites of the pENTR-3C plasmid (Thermo Fisher Scientific, Inc.), and then the pLenti6.3/V5-FHL1 plasmid $(3 \mu \mathrm{g})$ was constructed by EcoR I/Sal I restriction digestion and ligation (Takara Biotechnology Co., Ltd.). Next, the pLenti6.3/V5-FHL1 plasmid (3 $\mu \mathrm{g})$ was co-transfected with the packaging plasmid psPAX2 $(2.25 \mu \mathrm{g})$ and envelope plasmid pMD2.G $(0.75 \mu \mathrm{g})$ Promega Corporation) into 293T cells (ATCC). Following transfection by using Lipofectamine LTX $^{\text {TM }}$ (Invitrogen; Thermo Fisher Scientific, Inc.) for $48 \mathrm{~h}$, the supernatant was collected for the infection of the U87 cells, and then the stable FHL1 overexpression cell line was constructed through $1,000 \mu \mathrm{g} / \mathrm{ml}$ blasticidin selection. The sequences of the primers were as follows: FHL1, forward 5'-CATGGCGGA GAAGTTTGACTG-3' and reverse 5'-CCTATTGATGGTATA GGGCAGAAAG-3'; and $\beta$-actin, forward 5'-CTGGGACGA CATGGAGAAAA-3' and reverse 5'-GCCCAATACGACCAA ATCC-3'. $\beta$-actin was used as the internal control.

Next, a cell line with stable FHL1 knockdown was established. Small interfering RNA (siRNA) targeting the sequence of FHL1 and the control were inserted into the pLKO.1 vector (Addgene, Inc.), and the infection was performed following the recommended protocols (Shanghai GeneChem) by using Lipofectamine LTX $^{\mathrm{TM}}$ (Invitrogen; Thermo Fisher
Scientific, Inc.). Cells were seeded in 6-well plates at the density of $1 \times 10^{6}$ cells per well. Transfection was performed when cells have reached $\sim 80 \%$ confluence the next day by using Lipofectamine LTX $^{\mathrm{TM}}$. Briefly, $2 \mu \mathrm{g}$ plasmid and $5 \mu \mathrm{l}$ Lipofectamine LTX ${ }^{\mathrm{TM}}$ were mixed gently and incubated for $30 \mathrm{~min}$ at room temperature before transfection. The mixture was subsequently added to the cells at $37^{\circ} \mathrm{C}$. After $6 \mathrm{~h}$, culture medium was refreshed and cells were further cultured for $48 \mathrm{~h}$ before subsequent experiments. The sequence of siRNA targeting to FHL1 was AAGGAGGTGCACTATAAGAAC. The sequence of control siRNA was UUCUCCGAACGUGUC ACGUTT. The cell line then underwent $400 \mu \mathrm{g} / \mathrm{ml}$ puromycin selection. FHL1 overexpression or knockdown was confirmed by western blot analysis.

Reverse transcription (RT)-PCR. Total RNA was extracted from the 2 glioma U87 and U251 cell lines using TRIzol ${ }^{\circledR}$ reagent (Thermo Fisher Scientific, Inc.) quantified using NanoDrop $^{\mathrm{TM}}$ (Thermo Fisher Scientific, Inc.) and transcribed using a kit from Toyobo Life Science. RT-PCR and semi-quantitative PCR were performed by using a PrimerScript RT Reagent Kit (Takara Biotechnology Co., Ltd.) as previously described (24). $\beta$-actin was used as the internal control. Primer sequences were as follows: $\beta$-actin forward, CTGGGACGA CATGGAGAAAA; $\beta$-actin reverse, GCCCAATACGACCAA ATCC; FHL1 forward, CTGAAGTGCTTTGACAAGTTC; and FHL1 reverse, GTGCCAGTAGCGATTCTTAT.

Cell proliferation and cell cycle assay. Cell proliferation was analyzed using an MTT colorimetric assay (Sigma-Aldrich; Merck KGaA). Briefly, cells were counted and seeded in 4 96-well plates at a density of $2 \times 10^{3}$ cells/well. Each day, 1 plate was selected and $20 \mu \mathrm{l} \mathrm{MTT}(5 \mathrm{mg} / \mathrm{ml})$ was added to each well, and then the cells were incubated at $37^{\circ} \mathrm{C}$ for $4 \mathrm{~h}$. Following this, the medium was removed and $150 \mu 1$ dimethyl sulfoxide was added to solubilize the formazan precipitate. Absorbance was measured at $490 \mathrm{~nm}$ using a microplate reader. 
A

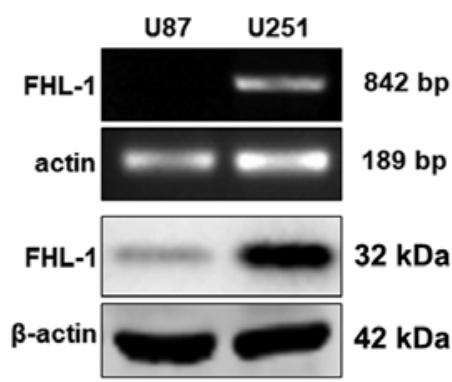

B

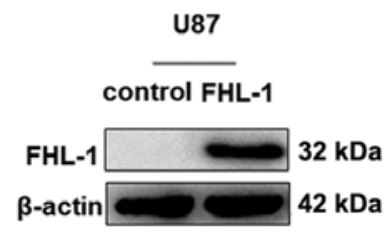

C

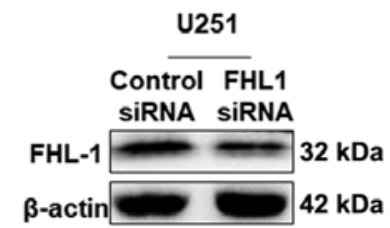

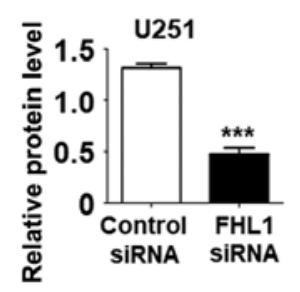

E
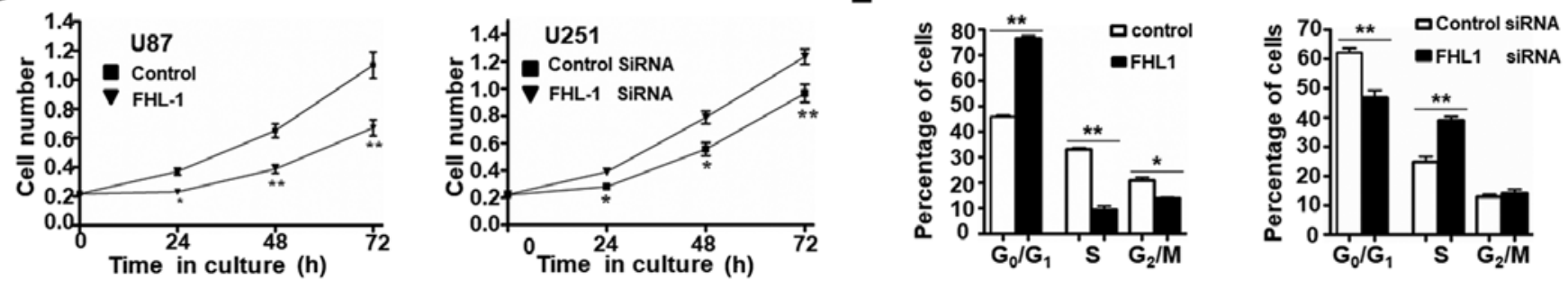

Figure 2. FHL1 inhibits the proliferation of glioma cells in vitro. (A) The mRNA and protein levels of FHL1 in 2 glioma U87 and U251 cell lines were examined using an reverse transcription polymerase chain reaction assay and western blot analysis, respectively. The expression of $\beta$-actin was used as an internal control. (B and C) The (B) stable FHL1-overexpressing U87 cell line and (C) stable FHL1-knockdown U251 cell line were confirmed by western blot analysis with anti-FHL1 antibody. These experiments were repeated 3 times. (D) Cell proliferation was examined every $24 \mathrm{~h}$ in the stable FHL-overexpressed U87 cells and stable FHL1-knockdown U251 cells using an MTT assay. (E) Cell cycle progression of 2 stable cell lines was determined by fluorescence-activated cell sorting following propidium iodide staining. The error bars represent the mean \pm standard deviation. ${ }^{*} \mathrm{P}<0.05,{ }^{* * *} \mathrm{P}<0.01$ and ${ }^{* * * *} \mathrm{P}<0.001$. FHL1, four-and-a-half LIM domains 1; siRNA, small interfering RNA.

The cell cycle analysis was performed as previously described (24). Briefly cells were resuspended in PBS and fixed in $70 \%$ ethanol for $2 \mathrm{~h}$. They were then stained with $20 \mathrm{mg} / \mathrm{ml}$ propidium iodide in PBS containing $0.1 \%$ Triton $\mathrm{X}-100$ and $0.2 \mathrm{mg} / \mathrm{ml}$ RNase A for $30 \mathrm{~min}$ on ice. The cells were the analyzed by a FACSCalibur flow cytometer (BD Immunocytometry Systems). The data were analyzed using CellQuest $^{\mathrm{TM}}$ Pro software (version 5.1; Becton, Dickinson and Company).

Co-immunoprecipitation (IP). PCR-amplified human FHL1 fragment was inserted into the multiple cloning sites of pCMV-FLAG vector and then confirmed by DNA sequencing. Cells were transfected with pCMV-FLAG-FHL1 or pCMV-FLAG using Lipofectamine ${ }^{\circledR}$ LTX \&Plus Reagent (Thermo Fisher Scientific, Inc.). Co-IP was performed as previously described (17), with an anti-FLAG monoclonal antibody (Merck KGaA; cat. no. F1804; 1:1,000.). Western blot analysis was performed using anti-FHL1 or anti-AKT antibody as aforementioned.

Statistical analysis. Statistical analysis was performed using Student's t-test and one-way analysis of variance with Least Significant Difference post hoc test, as appropriate. The association between FHL1 expression and clinicopathological variables was examined by Chi-square tests. The Kaplan-Meier method was used to calculate overall patient survival, while the difference in survival time between the two groups was examined using log-rank test. All P-values are two-tailed. $\mathrm{P}<0.05$ was considered to indicate a statistically significant difference. Data were analyzed using SPSS v.13.0 software (SPSS, Inc.).

\section{Results}

FHL1 expression in patients with glioma is negatively associated with prognosis. The expression of FHL1 was detected in 114 glioma specimens by immunohistochemistry. FHL1 was distributed in both the cytoplasm and the nucleus, but primarily in the cytoplasm. In all 114 glioma specimens, a high FHL1 expression was detected in low-grade glioma tissues while a low or no expression of FHL1 was detected in high-grade glioma tissues (Fig. 1A). No association was identified between FHL1 expression and sex, age, tumor position and extent of resection. However, FHL1 expression was negatively associated with histological grades (Table SI). The association between the survival time in patients with glioma following surgery and FHL1 expression was further analyzed. The data indicated that the low FHL1 expression was significantly associated with poor prognosis. When the expression of FHL1 was increased, the patients exhibited a longer survival time. These results suggested that the expression level of FHL1 in glioma was significantly associated with poor prognosis (Fig. 1B).

FHL1 inhibits glioma cell growth in vitro. Western blot analysis and RT-PCR were used to examine the expression level of FHL1 in 2 glioma cell lines (U87 and U251). The mRNA and protein expression levels of FHL1 were not detected in U87 cells, whereas FHL1 was highly expressed in U251 cells (Fig. 2A). As expected, FHL1 siRNAs specifically suppressed the endogenous expression of FHL1 in U251 cells and the overexpression of FHL1 also increased the protein level in U87 cells (Fig. 2B). The effects of FHL1 overexpression or knockdown of endogenous FHL1 protein on glioma cell growth were also investigated (Fig. 2C). MTT assay data indicated 
A
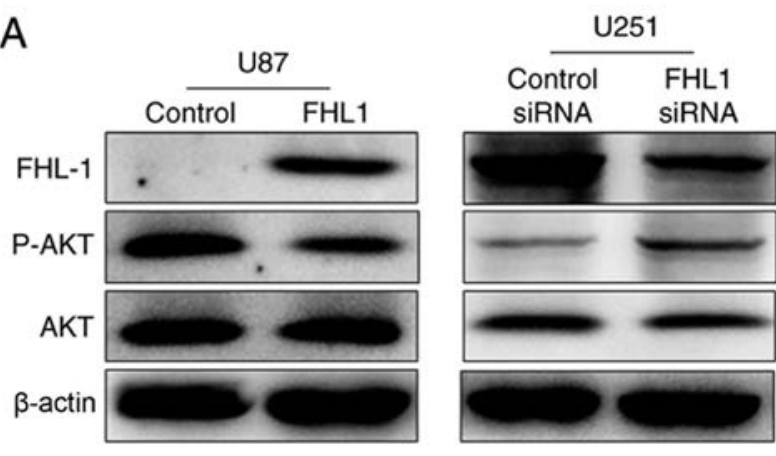

B

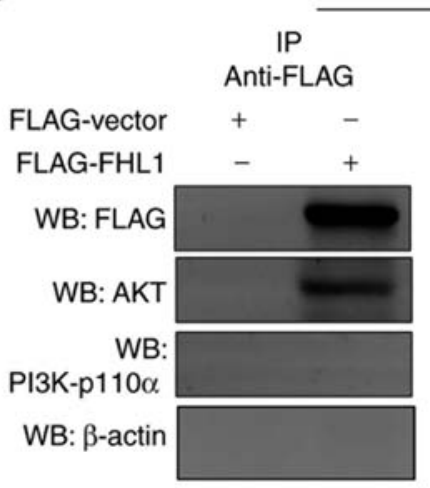

U87

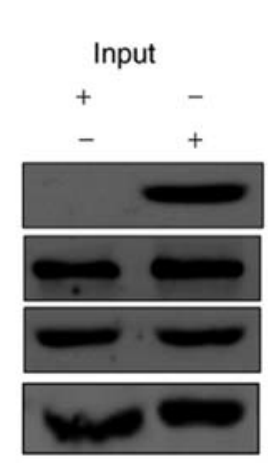

Figure 3. FHL1 affects glioma cell growth through PI3K/Akt signaling. (A) Western blot analysis for the phosphorylation levels of Akt and total AKT expression in the two types of stable cell lines and corresponding control cell lines, with $\beta$-actin as an internal control. (B) FHL1 interacts with AKT in U87 cells. U87 cells were transfected with $0.5 \mu \mathrm{g}$ FLAG-FHL1 expression vector or $0.5 \mu \mathrm{g}$ FLAG empty vector. The IP analysis was performed with anti-FLAG antibody, and the WB was performed with anti-AKT antibody and anti-p110 $\alpha$ antibody. FHL1, four-and-a-half LIM domains 1; p, phosphorylated; siRNA, small interfering RNA; IP, immunoprecipitation; WB, western blot analysis.

that U87 cells transfected with FHL1 expression vector grew more slowly compared with those transfected with the empty vector. By contrast, U251 cells transfected with FHL1 siRNAs grew at an increased rate compared with those transfected with control siRNA (Fig. 2D). The cell cycle assays indicated that FHL1 may inhibit cell growth by maintaining cells in the G0/G1 phase and FHL1 knockdown in U251 may promote cell proliferation by prompting cells into the $\mathrm{S}$ phase (Fig. 2E). These results suggested that FHL1 may inhibit glioma cell growth by maintaining cells in the $\mathrm{G}_{0} / \mathrm{G}_{1}$ phase.

FHL1 inhibits glioma growth by modulating PI3K/AKT signaling by interacting with $A K T$. Previous studies have indicated that PI3K/AKT signaling controls diverse cellular functions in gliomla, including proliferation, suggesting that it may serve a crucial role in modulating in the studies of glioma cell proliferation $(18,19)$. The phosphorylation levels of AKT at Thr308 were therefore detected by western blot analysis, which may significantly increase the activity of AKT protein. The expression levels of p-AKT were decreased in the FHL1-overexpressed U87 cell line, as compared with the control cells (Fig. 3A). By contrast, the expression levels of p-AKT were increased when FHL1 was knocked down in the U251 cell line, as compared with the control cells (Fig. 3A). In order to investigate the association between FHL1 and
PI3K/AKT signaling, Co-IP assays were performed to examine whether FHL1 may modulate PI3K/AKT signaling by interacting with kinase PI3K-p110 $\alpha$ or AKT. U87 cells were transfected with the FLAG-FHL1 expression or empty vectors, and Co-IP assays were performed with each antibody. The results demonstrated that FLAG-FHL1 interacted with AKT, subsequently preventing the binding of AKT and PI3K and further phosphorylation, as demonstrated by the lack of interaction between FLAG-FHL1 and PI3K (p110 $\alpha$ ). $\beta$-actin was considered as a randomized controlled protein and no detectable interaction was found between FLAG-FHL1 and $\beta$-actin (Fig. 3B). These data indicated that FHL1 inhibited glioma growth by modulating PI3K/AKT signaling by interacting with AKT.

FHL1 represses glioma growth in vivo through PI3K/AKT signaling. The effects of FHL1 on glioma growth in vivo were studied by subcutaneously injecting U87-FHL1 overexpression and U87 control stable cell lines. In the control group, the tumors gradually grew over time (Fig. 4A), suggesting that FHL1 overexpression may significantly inhibit tumor growth. The biggest tumor in the FHL1 overexpression group exhibited a volume of $499 \mathrm{~mm}^{3}$ (length=15.3 mm; width=8.00 $\mathrm{mm}$ ), however the biggest tumor in control group exhibited a volume of $198 \mathrm{~mm}^{3}$ (length=10.1 $\mathrm{mm}$; width=6.20 mm). (Fig. 4B and C). In addition, western blot analysis of the protein extracted from the subcutaneous transplantation of tumor tissues indicated that FHL1 overexpression was maintained in the transplanted tumors of the nude mice, and the levels of p-AKT (Thr308) expression decreased in transplanted tumor tissues formed by the U87-FHL1 overexpression cells, as compared with the control cells (Fig. 4D). The results indicated that FHL1 was responsible for the inhibition of transplanted tumor growth in nude mice by modulating $\mathrm{PI} 3 \mathrm{~K} / \mathrm{AKT}$ signaling through the decrease in phosphorylation at Thr308 of AKT.

\section{Discussion}

A growing body of evidence has demonstrated FHL1 to be significantly downregulated in various types of cancer, including hepatocellular carcinoma, as well as lung, bladder, gastric, prostate and breast cancer (6-13). In gastric and lung cancer, patients with a decreased expression of FHL1 suffer from significantly shorter survival times compared with those with increased FHL1 expression levels $(7,12)$. Furthermore, tumors with a decreased FHL1 expression level are generally more invasive $(11,12,15)$. Consistent with the data described for other types of cancer, the present study demonstrated for the first time, to the best of our knowledge, that gliomas with a decreased FHL1 expression level are more malignant compared with those with an increased FHL1 expression level, and that decreased FHL1 expression levels predicted shorter survival in patients with glioma.

FHL1 is regarded as a tumor suppressor by functions downstream of Src and Cas to suppress non-anchored tumor growth (6). In addition, FHL1 has been demonstrated to inhibit growth of various cancer cells by physically and functionally interacting with transcription factors. For example, Ding et al (8) demonstrated that FHL1 may inhibit 
A
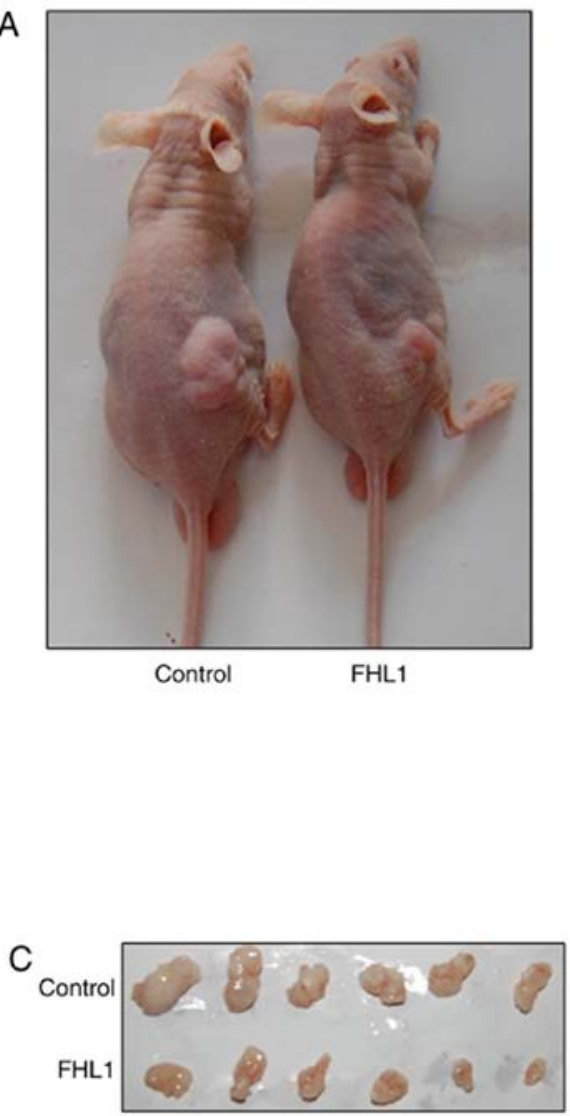

B

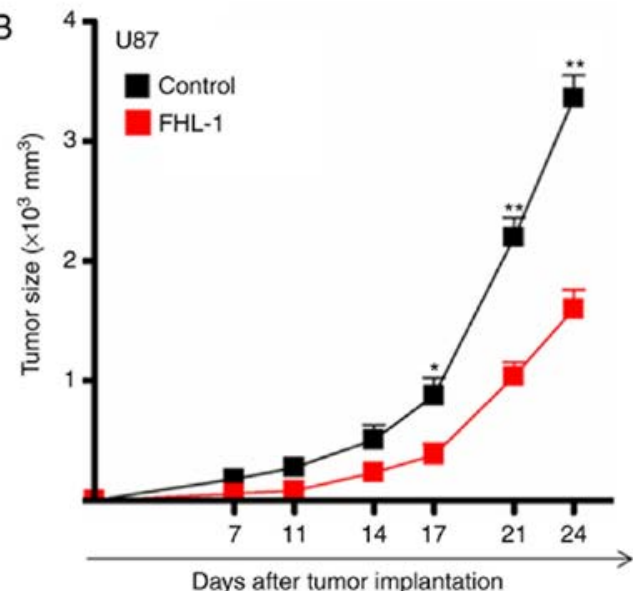

D

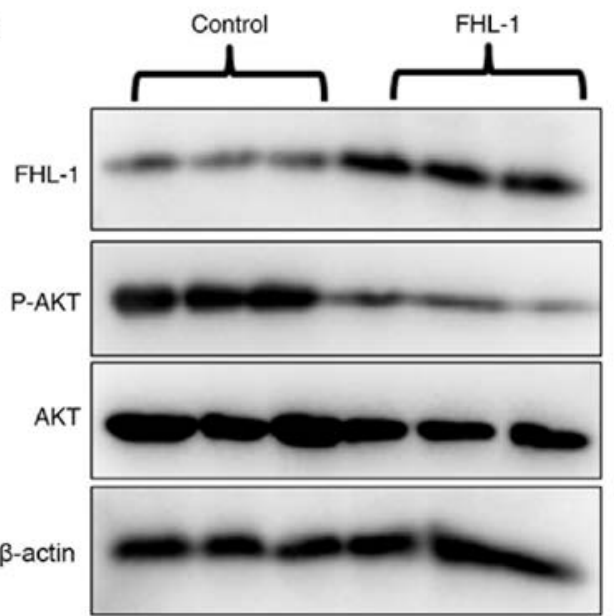

Figure 4. FHL1 overexpression inhibits tumor growth in vivo through PI3K/AKT signaling. (A) U87 cells stably transfected with FHL expression or control vector were inoculated subcutaneously in nude mice respectively $(n=6)$. (B) The tumor growth was monitored by measuring tumor size twice a week from the 7th day after tumor cell inoculation. (C) Tumors were dissected from mice on the last day of the experiments and images were captured. (D) Tumor tissue was lysed using lysis buffer, and the expression level of FHL1 and phosphorylated AKT (p-AKT) was analyzed by western blot analysis. Scale bars represent the mean \pm standard deviation. ${ }^{*} \mathrm{P}<0.05$ and ${ }^{* *} \mathrm{P}<0.01$. $\mathrm{n}=6$. FHL1, four-and-a-half LIM domains $1 ; \mathrm{p}$, phosphorylated.

hepatocellular carcinoma cell growth by interacting with Smad proteins, including Smad2-4, which are mediators of transforming growth factor- $\beta$ (TGF- $\beta$ ) signaling. The interaction between FHL1 and Smad complexes in the nucleus has been demonstrated to regulate TGF- $\beta$-responsive target gene transcription, including the activation of p21 and repression of the oncogene c-myc (8). Furthermore, Ding et al (10) also verified that FHL1 serves an important role in suppressing breast cancer cell growth by regulating estrogen receptor signaling and FHL1 interaction with ERs to repress the transcription of estrogen-responsive genes, trefoil factor 1 $(T F F 1)$ and cathepsin D (10). FHL1 can also interact with receptor-interacting protein of $140 \mathrm{kDa}$, a repressor for ER, to inhibit the transcription of the TFF1 gene in breast cancer cells (9) and FHL1 may downregulate estrogen receptor $\alpha$ activity by repressing AKT phosphorylation in human breast cancer cells. It should be noted that the human glioblastoma U251 cell line is PTEN-deficient and exhibits high level of phosphorylated AKT (25). In the present study, we hypothesized that the interaction between FHL1 with AKT may affect the spatial structure of AKT and decrease, at least partially, the phosphorylation efficiency of AKT by PI3K. Therefore, following disruption of the binding of FHL1 and AKT by FHL1 silencing, normal PI3K/AKT signaling was restored and activated, as demonstrated by the increased levels of p-AKT in FHL1-knockdown U251 cells. A recent study by Niu et al (12) indicated that FHL1 may also inhibit lung cancer cell growth by markedly inhibiting the expression of cyclin A, B1 and D and inducing the expression of cyclin-dependent kinase inhibitors p21and p27, which lead to G1 and the G2/M cell cycle arrest. Similarly, the present study identified that FHL1 may inhibit glioma cell proliferation in vitro and impede tumorigenesis in vivo.

Previous studies have indicated that PI3K/AKT signaling affects cell growth, proliferation and survival in numerous types of cancer, including glioma (26). The inactivation of $\mathrm{PI} 3 \mathrm{~K} / \mathrm{AKT}$ signaling inhibits glioma cell growth (27). In addition, Cyr61-modulated hepatocyte growth factor-dependent tumor cell growth is regulated by AKT (28), which results in PI3K/AKT signaling as the principal target in the studies of glioma cell proliferation. The present study also demonstrated that FHL1 inhibits proliferation in vivo and in vitro through its 
interaction with AKT, following decreased phosphorylation at Thr308 of AKT. However, only co-immunoprecipitation has been performed to demonstrate the interaction between FHL1 and AKT. Additional experiments are required for further functional studies.

The present study indicated that FHL1 may be a tumor suppressor in glioma. Firstly, FHL1 was downregulated in high-grade glioma tissues with more malignant biological behaviors, as compared with low-grade glioma tissues. Secondly, the downregulation of FHL1 predicted shorter survival in patients with glioma. Thirdly, the overexpression of FHL1 suppressed cell growth in human glioma cell lines and endogenous FHL1 knockdown promoted glioma cell growth. It was also identified that FHL1 may inhibit glioma cell growth in nude mice. Finally, the mechanism of action of the inhibitory effect of FHL1 on the proliferation of glioma cells was demonstrated to function through the regulation of $\mathrm{PI} 3 \mathrm{~K} / \mathrm{AKT}$ signaling, through direct interaction with AKT. Therefore, these data suggested that FHL1 may serve an important role in the development and progression of glioma, and that FHL1 may be a new diagnostic and prognostic marker and novel target for glioma treatment in the future.

\section{Acknowledgements}

Not applicable.

\section{Funding}

The present study was supported by The National Natural Science Foundation of China (grant nos. 81402438, 81502145 and 81071874) and Natural Science Foundation of Shaanxi (grant nos. 2016JQ8017 and 2019SF117).

\section{Availability of data and materials}

The datasets used and/or analyzed during the current study are available from the corresponding author on reasonable request.

\section{Authors' contributions}

SZL performed immunohistochemical staining of the clinical samples, interpreted the patient data regarding the grade and prognosis of patients with glioma and performed animal experiments. YYH performed western blotting and RT-PCR and analysed the corresponding data. JLZ performed lentiviral packaging, cell proliferation and cell cycle assays, and co-immunoprecipitation. JZ performed statistical analysis for clinical data and analyzed the corresponding data. HYQ was a major contributor in conception and design of this study. $\mathrm{HH}$ analysed and interpreted the whole data of this study and drafted the manuscript. $\mathrm{ZF}$ participated in the whole process of the present study, including the study design, experiment guide, data interpretation and manuscript revision. All of the authors participated sufficiently in the work to take public responsibility for appropriate portions of the content and agreed to be accountable for all aspects of the work in ensuring that questions related to the accuracy or integrity of any part of the work are appropriately investigated and resolved. All authors read and approved the final manuscript.

\section{Ethics approval and consent to participate}

The animal studies were approved by the Animal Experiment Administration Committee of the Fourth Military Medical University (approval no., 20190211), and in accordance with the recommendations of Guide for the Care and Use of Laboratory Animals prepared by the National Academy of Sciences and published by the National Institutes of Health (NIH publication 86-23, revised 1985). All patients involved in the study provided written informed consent for the use of their samples, and the protocols involving human samples were approved by the Ethics Committee of Xijing Hospital, Fourth Military Medical University (approval no. KY20183175-1.)

\section{Patient consent for publication}

Not applicable.

\section{Competing interests}

The authors declare that they have no competing interests.

\section{References}

1. Hegi ME, Diserens AC, Gorlia T, Hamou MF, de Tribolet N, Weller M, Kros JM, Hainfellner JA, Mason W, Mariani L, et al: MGMT gene silencing and benefit from temozolomide in glioblastoma. N Engl J Med 352: 997-1003, 2005.

2. Stupp R, Hegi ME, Mason WP, Van den Bent MJ, Taphoorn MJ, Janzer RC, Ludwin SK, Allgeier A, Fisher B, Belanger K, et al: Effects of radiotherapy with concomitant and adjuvant temozolomide versus radiotherapy alone on survival in glioblastoma in a randomised phase III study: 5-year analysis of the EORTC-NCIC trial. Lancet Oncol 10: 459-466, 2009.

3. Lee SM, Tsui SK, Chan KK, Garcia-Barcelo M, Waye MM, Fung KP, Liew CC and Lee CY: Chromosomal mapping, tissue distribution and cDNA sequence of four-and-a-half LIM domain protein 1 (FHL1). Gene 216: 163-170, 1998.

4. Bach I: The LIM domain: Regulation by association. Mech Dev 91: 5-17, 2000.

5. Cowling BS, McGrath MJ, Nguyen MA, Cottle DL, Kee AJ, Brown S, Schessl J, Zou Y, Joya J, Bönnemann CG, et al: Identification of FHL1 as a regulator of skeletal muscle mass: Implications for human myopathy. J Cell Biol 183: 1033-1048, 2008.

6. Shen Y, Jia Z, Nagele RG, Ichikawa H and Goldberg GS: SRC uses Cas to suppress Fhl1 in order to promote nonanchored growth and migration of tumor cells. Cancer Res 66: 1543-1552, 2006.

7. Sakashita K, Mimori K, Tanaka F, Kamohara Y, Inoue H, Sawada T, Hirakawa K and Mori M: Clinical significance of loss of Fhl1 expression in human gastric cancer. Ann Surg Oncol 15: 2293-2300, 2008.

8. Ding L, Wang Z, Yan J, Yang X, Liu A, Qiu W, Zhu J, Han J, Zhang H, Lin J, et al: Human four-and-a-half LIM family members suppress tumor cell growth through a TGF-beta-like signaling pathway. J Clin Invest 119: 349-361, 2009.

9. Lin J, Ding L, Jin R, Zhang H, Cheng L, Qin X, Chai J and Ye Q: Four and a half LIM domains 1 (FHL1) and receptor interacting protein of $140 \mathrm{kDa}$ (RIP140) interact and cooperate in estrogen signaling. Int J Biochem Cell Biol 41: 1613-1618, 2009.

10. Ding L, Niu C, Zheng Y, Xiong Z, Liu Y, Lin J, Sun H, Huang K, Yang W, Li X and Ye Q: FHL1 interacts with oestrogen receptors and regulates breast cancer cell growth. J Cell Mol Med 15: 72-85, 2011.

11. Matsumoto M, Kawakami K, Enokida H, Toki K, Matsuda R, Chiyomaru T, Nishiyama K, Kawahara K, Seki N and Nakagawa M: CpG hypermethylation of human four-and-a-half LIM domains 1 contributes to migration and invasion activity of human bladder cancer. Int J Mol Med 26: 241-247, 2010. 
12. Niu C, Liang C, Guo J, Cheng L, Zhang H, Qin X, Zhang Q, Ding L, Yuan B, Xu X, et al: Downregulation and growth inhibitory role of FHL1 in lung cancer. Int J Cancer 130: 2549-2556, 2011

13. Zhang F, Feng F, Yang P, Li Z, You J, Xie W, Gao X and Yang J: Four-and-a-half-LIM protein 1 down-regulates estrogen receptor $\alpha$ activity through repression of AKT phosphorylation in human breast cancer cell. Int J Biochem Cell Biol 44: 320-326, 2012.

14. Xu Y, Liu Z and Guo K: Expression of FHL1 in gastric cancer tissue and its correlation with the invasion and metastasis of gastric cancer. Mol Cell Biochem 363: 93-99, 2012.

15. Li X, Jia Z, Shen Y, Ichikawa H, Jarvik J, Nagele RG and Goldberg GS: Coordinate suppression of Sdpr and Fhll expression in tumors of the breast, kidney, and prostate. Cancer Sci 99: 1326-1333, 2008.

16. Cao W, Liu J, Xia R, Lin L, Wang X, Xiao M, Zhang C, Li J, Ji T and Chen W: X-linked FHL1 as a novel therapeutic target for head and neck squamous cell carcinoma. Oncotarget 7: 14537-14550, 2016.

17. Wang X, Wei X, Yuan Y, Sun Q, Zhan J, Zhang J, Tang Y, Li F, Ding L, Ye Q and Zhang H: Src-mediated phosphorylation converts FHL1 from tumor suppressor to tumor promoter. J Cell Biol 217: 1335-1351, 2018.

18. Dey N, Crosswell HE, De P, Parsons R, Peng Q, Su JD and Durden DL: The protein phosphatase activity of PTEN regulates SRC family kinases and controls glioma migration. Cancer Res 68 $1862-1871,2008$

19. Nakamura JL, Garcia E and Pieper RO: S6K1 plays a key role in glial transformation. Cancer Res 68: 6516-6523, 2008.

20. Li Y, Ma X, Wang Y and Li G: miR-489 inhibits proliferation, cell cycle progression and induces apoptosis of glioma cells via targeting SPIN1-mediated PI3K/AKT pathway. Biomed Pharmacother 93: 435-443, 2017.

21. Nan Y, Guo L, Song Y, Wang L, Yu K, Huang Q and Zhong Y: Combinatorial therapy with adenoviral-mediated PTEN and a PI3K inhibitor suppresses malignant glioma cell growth in vitro and in vivo by regulating the PI3K/AKT signaling pathway. J Cancer Res Clin Oncol 143: 1477-1487, 2017.
22. Bayne K: Revised Guide for the Care and Use of Laboratory Animals available. American Physiological Society. Physiologist 39: 199, 208-211, 1996

23. Louis DN, Ohgaki H, Wiestler OD, Cavenee WK, Burger PC, Jouvet A, Scheithauer BW and Kleihues P: The 2007 WHO classification of tumours of the central nervous system. Acta Neuropathol 114: 97-109, 2007.

24. Wang H, Yan X, Ji LY, Ji XT, Wang P, Guo SW and Li SZ: miR-139 functions as an antioncomir to repress glioma progression through targeting IGF-1 R, AMY-1, and PGC-1 $\beta$. Technol Cancer Res Treat 16: 497-511, 2017.

25. Wang YT, Yuan B, Chen HD, Xu L, Tian YN, Zhang A, He JX and Miao ZH: Acquired resistance of phosphatase and tensin homolog-deficient cells to poly(ADP-ribose) polymerase inhibitor and Ara-C mediated by 53BP1 loss and SAMHD1 overexpression. Cancer Sci 109: 821-831, 2018.

26. Vivanco I and Sawyers CL: The phosphatidylinositol 3-Kinase AKT pathway in human cancer. Nat Rev Cancer 2: 489-501, 2002.

27. Han L, Yang Y, Yue X, Huang K, Liu X, Pu P, Jiang H, Yan W, Jiang $\mathrm{T}$ and Kang $\mathrm{C}$ : Inactivation of PI3K/AKT signaling inhibits glioma cell growth through modulation of $\beta$-catenin-mediated transcription. Brain Res 1366: 9-17, 2010.

28. Goodwin CR, Lal B, Zhou X, Ho S, Xia S, Taeger A, Murray J and Laterra J: Cyr61 mediates hepatocyte growth factor-dependent tumor cell growth, migration, and Akt activation. Cancer Res 70: 2932-2941, 2010.

(i) $\Theta$ This work is licensed under a Creative Commons Attribution-NonCommercial-NoDerivatives 4.0 International (CC BY-NC-ND 4.0) License. 